\title{
ANÁLISE DA ESPESSURA DOS MÚSCULOS MASTIGATÓRIOS POR MEIO DE TOMOGRAFIA COMPUTADORIZADA HELICOIDAL
}

\section{ANALYSIS OF MASTICATORY MUSCLES USING HELICAL COMPUTED TOMOGRAPHY}

\author{
Daniela de Paula Lica* \\ Maria José Albuquerque Pereira de Sousa e Tucunduva**
}

\section{RESUMO}

A análise da espessura dos músculos mastigatórios pela tomografia computadorizada (TC) mostra-se importante para estabelecer parâmetros de normalidade e, assim, diferirem-se os padrões de normalidade de afecções e distúrbios relacionados à mastigação. O sistema mastigatório depende de um delicado equilíbrio entre a musculatura, a estrutura óssea e os dentes, no qual qualquer interferência ou doença associada a um de seus componentes resultam em desequilíbrio de todo o sistema. Este estudo buscou analisar a simetria bilateral dos músculos mastigatórios, contando com uma amostra de 72 exames de TC obtidos por banco de dados (CEP n.13668469). Foram realizadas as mensurações nos cortes axial e coronal. Foram calculadas as médias para cada m. mastigatório, podendo-se fazer comparações entre o lado esquerdo e direito dos pares de músculos e correlacionar a espessura muscular com a espessura da cabeça da mandíbula. Concluiu-se que não houve simetria bilateral, porém as médias obtidas com relação à espessura dos músculos tiveram uma diferença pequena. O músculo que obteve a maior média de volume foi o $\mathrm{m}$. pterigóideo lateral direito e o $\mathrm{m}$. que obteve a menor média de volume foi o m. temporal esquerdo. Quanto à correlação entre espessura da cabeça da mandíbula e espessura dos músculos mastigatórios, houve um equilíbrio entre os valores, ou seja, a musculatura é mais espessa em indivíduos com a cabeça da mandíbula mais espessa assim como os indivíduos que obtiveram os menores valores.

DESCRITORES: Músculos mastigatórios • Tomografia computadorizada por Raios X • Diagnóstico por imagem

\section{ABSTRACT}

The analysis of masticatory muscles using computed tomography (CT) is important to establish normal parameters and thus differ from a range of conditions and disorders related to chewing. The masticatory system depends on a delicate balance between muscles, teeth and bone structures in which, when there is any interference or disease associated with its components, this may result in an imbalance of the whole system. This study is aimed to analyze the bilateral symmetry of the masticatory muscles through a sample of 72 CT scans obtained by database (CEP n.13668469). Measurements were performed in the axial and coronal scans. Averages were calculated for each masticatory muscle and comparisons between the left and right pairs of muscles and muscle thickness correlated with the dimensions of the condyle. It was concluded that there was bilateral symmetry, however the mean values to the thickness of the muscles werw slightly differante. The muscle whith the highest volume average was the right lateral pterygoid muscle and the lowest average volume was the left temporal muscle. Regarding the correlation between the condyle dimension and masticatory muscles, there was a balance between the values, that is, the thicker the muscle the greater the condyle DESCRIPTORS: Masticatory muscles • Tomography, X-ray computed $\bullet$ Diagnostic imaging 


\section{N T R O D U Ç Ã 0}

São considerados quatro músculos pertencentes ao grupo da mastigação: três elevadores, masseter, temporal e pterigóideo medial e um protrusor da mandíbula, m. pterigóideo lateral. Todos eles ligam a mandíbula ao crânio, isto é, tomam origem no crânio (ponto fixo) e inserem-se na mandíbula (ponto móvel) ${ }^{1}$.

A articulação temporomandibular (ATM) é um sistema articular extremamente complexo. Cada uma das articulações pode, ao mesmo tempo, agir de forma diferente e separadamente, mas não completamente sem alguma influência de uma na outra. Um entendimento completo da biomecânica da ATM é essencial e básico para o estudo de função e disfunção do sistema mastigatório ${ }^{2}$.

A TC é o exame ideal para análise das estruturas do sistema mastigatório por reduzir sobremaneira a sobreposição de imagens ${ }^{3}$.

\section{OB JET I V 0}

Analisar a espessura dos m.m. mastigatórios por meio de imagens de TC, buscando possível simetria bilateral e correlacionar a espessura dos m.m. com a espessura da cabeça da mandíbula.

\section{METODOLOGIA}

Foram utilizados 72 exames de TC de banco de dados (CEP n.13668469). A mensuração foi realizada no programa ImageJ em imagens com cortes axial e sagital.

\section{REVISÃO DE LITERATURA}

O m. temporal tem uma ampla origem na superfície lateral do crânio e estendese para frente até a borda lateral da crista supraorbital. A inserção é no processo coronoide e ao longo do bordo anterior do ramo da mandíbula. As fibras anteriores são verticais, as fibras médias têm curso oblíquo, e as posteriores são horizontais antes de se dobrarem para baixo em direção à mandíbula. A inervação do m. temporal é normalmente feita por três ramos do nervo temporal, da divisão mandibular do nervo trigêmeo, $V$ par craniano ${ }^{4}$.

$\mathrm{O} \mathrm{m}$. masseter tem forma aproximada- mente retangular e é formado por dois feixes musculares que se estendem do arco zigomático ao ramo mandibular. Sua inserção estende-se da região do segundo molar na superfície externa da mandíbula ao terço inferior da superfície lateral posterior do ramo ${ }^{4}$.

O m. pterigóideo medial é um m. retangular com sua origem principalmente na fossa pterigóidea e sua inserção na superfície medial do ângulo da mandíbula. O músculo, a partir da sua origem, corre para baixo, posterior e lateralmente a sua inserção ${ }^{4}$.

O m. pterigóideo lateral tem dois feixes: um maior, chamado feixe inferior, que se origina na superfície externa da lateral da lâmina pterigóidea, e um menor, chamado feixe superior que se origina da asa maior do osso esfenoide. Ambas as divisões juntam-se em frente da ATM próximo ao processo condilar. Ambos os feixes inserem-se na fóvea pterigóidea com uma variável para o feixe superior que se insere no disco articular e cápsula. As fibras mais anteriores do feixe superior do m. entrelaçam-se com as fibras mais profundas do m. temporal, pelo fato de que ambos emergem do limite anterior do teto da fossa infratemporal. Algumas fibras do feixe superior inserem-se na fóvea pterigóidea do pescoço do côndilo e no disco articular. Fibras do m. pterigóideo lateral são entrelaçadas lateralmente por fibras do $\mathrm{m}$. pterigóideo medial ${ }^{4}$.

O aparelho estomatognático depende de um delicado equilíbrio para a correta execução de suas funções miofuncionais e orofaciais, ou seja, mastigação, deglutição e respiração. Contudo, tal equilíbrio poderia ser quebrado por desarmonias nas estruturas tanto ósseas quanto dentais. Esse desequilíbrio causaria interferência intimamente na qualidade de vida do paciente em âmbitos funcionais, estéticos, psicológicos e sociais. Os indivíduos que possuem desproporções maxilomandibulares apresentam características singulares quanto à adaptação dos músculos, viabilizando a melhor realização das funções do aparelho estomatognático de acordo com o padrão das bases ósseas do esqueleto ${ }^{5}$.

Disfunção temporomandibular (DTM) é um conjunto de sinais e sintomas entre $\angle I C A \quad D P$

TUCUNDUVA MJAPS

ANÁLISE DA

ESPESSURA

DOS MÚSCULOS

MASTIGATÓRIOS

POR MEIO DE

TOMOGRAFIA

COMPUTADORIZADA

HELICOIDAL
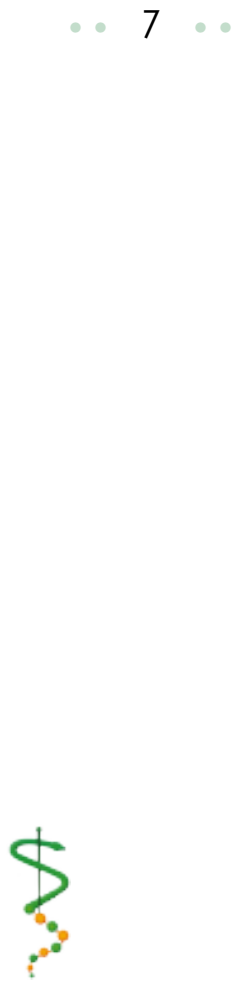

REV, ODONTOL.

Univ. CID. São

Paulo

2014; 26(1): 188 -

95, JAN-ABR 
$\angle I C A \quad D P$

TUCUNDUVA MJAPS

ANÁLISE DA

ESPESSURA

DOS MÚSCULOS

MASTIGATÓRIOS

POR MEIO DE

TOMOGRAFIA

COMPUTADORIZADA

HELICOIDAL

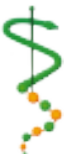

REV, ODONTOL.

UNIV, CID, SÃO

PAULO

2014; 26(1): 6-12,

JAN - ABR
I SSN 1983-5183

os quais estão dor nos m.m. mastigatórios, dificuldade de movimentação mandibular, ruídos na articulação e sintomas auditivos. A DTM pode ser diagnosticada apenas quando há uma somatória desses sinais e sintomas, pois um deles isoladamente não define se o paciente é portador de DTM. O cirurgião-dentista tem importante papel nas equipes de tratamento e empregam a modalidade terapêutica da placa de oclusão ou miorrelaxante e o objetivo desta é simular uma oclusão normal e proteger os elementos dentais dos pacientes portadores de bruxismo ${ }^{6}$. Desordem temporomandibular: análise da freqüência e severidade dos sinais e sintomas antes e após a placa de oclusão ${ }^{7}$.

As DTMs referem-se a distúrbios que envolvam os m.m. mastigatórios, a ATM ou qualquer um de seus elementos constituintes. Caracterizam-se por dores orofaciais e limitação da movimentação mandibular. Possuem prevalência variável, dependendo se é única ou se há uma somatória de fatores ${ }^{7}$.

Castelo et al. ${ }^{8}$ (2007) realizaram uma pesquisa visando a mensuração dos $\mathrm{m}$. mastigatórios em crianças com mordida cruzada posterior unilateral na dentição decídua e mista inicial por meio de ultrassonografia. A amostra consistiu de 49 crianças de ambos os gêneros. Os resultados obtidos mostraram que nos grupos de dentição decídua normal (DN) e com mordida cruzada (DC) não houve diferença significativa na espessura muscular entre os lados, tanto em máxima intercuspidação, como no repouso. Na dentição mista inicial, tanto na oclusão normal $(\mathrm{MN})$ como na mordida cruzada (MC), não houve diferença na espessura do $\mathrm{m}$. masseter entre os lados, em ambas as posições mandibulares. Já no grupo $M C, a$ porção anterior do temporal do lado cruzado apresentou espessura estatística e significativamente maior em relação ao lado normal no repouso $(\mathrm{p}<0,05)^{8}$.

A eletromiografia vem sendo utilizada para a avaliação na clínica odontológica em pacientes que reclamam de dores ou incômodos relacionados aos m. mastigatórios e cefaleias tensionais. Duarte-Kroll et al. ${ }^{9}$ (2010) desenvolveram um estudo utilizando-se de 41 indivíduos do gêne- ro feminino, entre 18 e 45 anos de idade, que não possuíam doenças sistêmicas como artrite, artrose, diabetes e patologias neurológicas. 20 eram sintomáticos e 21 assintomáticos para DTM. Durante a avaliação eletromiográfica, os m.m. masseteres apresentaram menor amplitude no grupo DTM do que no grupo-controle. Nesta pesquisa, realizou-se apenas uma repetição da mastigação habitual para a avaliação do sinal eletromiográfico, na tentativa de ser o mais fiel possível a uma situação clínica. Na medida em que isso afeta os modelos de contração, consequentemente, a confiabilidade dos registros mensurados durante a mastigação fica comprometida. A partir da metodologia utilizada, bem como dos resultados obtidos, é possível sugerir que os dados absolutos podem comprovar achados clínicos observados na análise qualitativa do sinal eletromiográfico ${ }^{9}$.

Botelho et al. ${ }^{10}$ (2008) realizaram um estudo buscando analisar a assimetria de pares de m.m. mastigatórios de sujeitos saudáveis com oclusão normal, a fim de fornecer dados que podem servir de parâmetros em futuras comparações com sujeitos portadores de DTM, distinguindo, portanto um desequilíbrio muscular normal de um patológico, auxiliando o diagnostico desta. O objetivo foi descrever os parâmetros de assimetria dos m.m. temporal e masseter. Participaram trinta sujeitos, todos saudáveis e apresentando dentição permanente completa e sadia, incluindo os segundos molares, com relação molar e canino em classe I de Angle bilateral (+/- $1 \mathrm{~mm})$, bem como overjet $\mathrm{e}$ overbite variando entre 2 e $5 \mathrm{~mm}$. A avaliação foi realizada por inspeção visual. Os sujeitos ficaram sentados numa cadeira com encosto sem apoio de cabeça e com os pés apoiados no chão. As ondas eletromiográficas foram comparadas entre os lados direito e esquerdo dos pares musculares, computando-se o coeficiente de porcentagem de sobreposição (POC, \%). Além disso, foi calculado o coeficiente de torque (TC, \%), que avalia o componente de deslocamento lateral da mandíbula quando há contração desequilibrada entre masseteres e temporais contralaterais. As médias e desvios-padrão foram: POC $\mathrm{t}$ : 
$86,96 \pm 3,20 ;$ POC m: 86,51 $\pm 3,33$; POC médio: $86,75 \pm 2,48$; TC: $8,60 \pm 1,08$. Os resultados demonstraram que os sujeitos adultos jovens avaliados apresentaram valores médios de assimetria dentro dos padrões de normalidade já estabelecidos previamente para outras populações. Esse padrão de normalidade parece refletir num bom desempenho funcional do sistema estomatognático, como o encontrado na avaliação miofuncional ${ }^{10}$.

A mastigação tem sido um assunto amplamente abordado na literatura, isso porque é de extrema importância ao sistema digestório e possui íntima relação com o aparelho estomatognático. Existem fatores como capacidade articular e muscular e saúde das estruturas orofaciais que são indispensáveis para determinar o tipo e a qualidade da mastigação do indivíduo. Grupos de portadores de DTM têm sido comparados com grupos-controle a fim de se demonstrarem as limitações na função mastigatória. Esta pode ser afetada por uma complexa interação de efeitos diretos e indiretos, podendo cada um deles ser estudado e pesquisado de maneira isolada. Felício et al. ${ }^{11}$ (2007) obtiveram uma amostra composta por 20 sujeitos com DTM e 10 como grupo-controle que foram selecionados com anamnese e exame clínico, responderam a questões sobre sua autopercepção de severidade de dor e sons nas ATMs, dor nos músculos, entre outros sintomas. Submeteram-se a exame clínico, considerando-se o número de elementos dentais presentes e a análise funcional da oclusão - medidas de abertura oral, excursão lateral da mandíbula, contatos oclusais do lado de trabalho e balanceio, entre outros. A mastigação foi avaliada quanto ao tempo de ingestão, número de golpes e tipo mastigatório (uni ou bilateral) usando-se um biscoito recheado, cuja força máxima para quebrá-lo no primeiro momento foi de 4341,8g, como verificado com o auxílio do Texture Analyser TA-XT2 (Stable Micro Systems). Para a comparação entre os grupos foi realizada a análise de variância, considerando-se os grupos como variáveis dependentes e os outros fatores como variáveis independentes. $\mathrm{O}$ teste de correlação produto-momento de Pearson foi aplicado para investigar as possíveis correlações entre as variáveis. Resultou-se que a maioria dos sujeitos de grupo-controle apresentou tipo de mastigação bilateral enquanto o grupo DTM mostrou tendência ao tipo unilateral. O tempo e o tipo mastigatório foram correlacionados, respectivamente de modo positivo e negativo, à severidade da DTM e ao número de interferências oclusais. No grupo com DTM a mastigação diferiu do padrão fisiológico normal. O número de interferências oclusais e a severidade da DTM foram as variáveis correlacionadas à mastigação ${ }^{11}$.

D'Ippolito et al. ${ }^{12}$ (2010) promoveram um estudo para avaliar a visibilidade do m. pterigóideo lateral (MPL) na ATM, utilizando-se de imagens obtidas por ressonância magnética, por meio de diferentes projeções, e compararam os achados de imagem com sintomas clínicos de pacientes com e sem desordens temporomandibulares (DTM). As imagens em diferentes projeções de 100 ATMs de 35 participantes (70 ATMs) e com DTM,1 5 participantes (30 ATMs) sem sinais clínicos e sintomas de DTM visíveis e analisados. Os m.m. mastigatórios estão frequentemente relacionados à DTM, porque, uma vez que recebem sobrecarga geralmente causada por habitos parafuncionais e desordens oclusais, sua manifestação clínica de dor é traduzida. O MPL, um dos mais importantes músculos da fisiologia da mastigação, está diretamente ligado à ATM e participa nos movimentos de abertura, fechamento, de lateralidade, mandibular e protrusão. A ressonância magnética é um tipo de exame altamente eficiente para a avaliação de alterações de ATM e sua musculatura, porém existem poucos trabalhos que se dedicam a avaliar esssas condições no MPL, tais como contratura hipertrófica, atrofia muscular associada ou não com o disco articular. O grupo de estudo consistiu de 50 participantes (100 ATMs), 38 mulheres e 12 homens, tendo sido subdividido em pacientes sintomáticos (35 indivíduos) e 15 indivíduos assintomáticos voluntários, como controle. A dor miofascial, limitação da abertura da boca, sons articulares e bruxismo foram considerados como sintomas clínicos de DTM. Todos os pacientes foram submetidos a exame de resso- $\angle I C A \quad D P$

TUCUNDUVA MJAPS

ANÁLISE DA

ESPESSURA

DOS MÚSCULOS

MASTIGATÓRIOS

POR MEIO DE

TOMOGRAFIA

COMPUTADORIZADA

HELICOIDAL

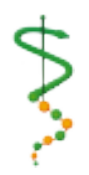

REV, ODONTOL.

UNIV, CID, São

PAULO

2014; 26(1): 188 -

95, JAN-ABR 
$\angle I C A \quad D P$ TUCUNDUVA MJAPS

ANÁLISE DA ESPESSURA

DOS MÚSCULOS MASTIGATÓRIOS

POR MEIO DE TOMOGRAFIA

COMPUTADORIZADA HELICOIDAL

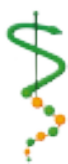

REV. ODONTOL UNIV, CID, SÃO PAULO 2014; 26(1): 6-12, $J A N-A B R$ nância magnética bilateral da ATM. Após a obtenção das imagens, as medições do MPL foram realizadas utilizando-se uma estação de trabalho, nos cortes axial e sagital, ponderadas em T1 e T2, com a boca aberta e fechada, respectivamente, e foram selecionados porque os músculos se tornaram mais visíveis e representaram diferentes momentos durante o exame. Para determinar se as diferenças entre as medidas foram estatisticamente significativas, foram comparados os valores MPL em cada sequência de imagens de RM dos lados direito e esquerdo do mesmo paciente, e dos grupos com e sem DTM. Pôde-se concluir que, na presença de DTM, houve alterações na espessura do MPL, sendo as principais alterações a atrofia muscular e a contratura ${ }^{12}$.

\section{RESULTADOS}

A média encontrada para o m. pterigóideo medial direito foi o valor de $52,8 \mathrm{~mm}$. O maior valor encontrado na mensuração das amostras foi de $79 \mathrm{~mm}$ e o menor foi de $35 \mathrm{~mm}$. A média encontrada para o m. pterigóideo medial esquerdo foi de $49,2 \mathrm{~mm}$. O maior valor encontrado na amostra mensurada foi de $78 \mathrm{~mm}$ e o menor $27 \mathrm{~mm}$. Houve diferença na média de 3,6mm comparando-se o m. pterigóideo medial esquerdo e direito, sendo que é o grupo que apresenta o maior valor de diferença obtida entre as médias encontradas. A média encontrada para o m. pterigóideo lateral direito resultou em $70,6 \mathrm{~mm}$. Sendo o maior valor encontrado $94 \mathrm{~mm}$ e o menor $42 \mathrm{~mm}$. Já a média para o $\mathrm{m}$. pterigóideo lateral esquerdo ficou em $69,7 \mathrm{~mm}$. O maior valor desta parte da amostra foi de $103 \mathrm{~mm}$ e o menor $41 \mathrm{~mm}$. Há uma diferença de 0,9mm comparando-se as médias do m. pterigóideo lateral lado esquerdo com o direito; este grupo foi o que apresentou menor diferença de médias comparando-se com os demais pares de m.m. mastigatórios. A média conseguida pela amostra mensurada para o m. masseter do lado direito foi de $44,0 \mathrm{~mm}$, sendo que o maior valor obtido foi de $64 \mathrm{~mm}$ e o menor $21 \mathrm{~mm}$. Foi conseguida a média de $45,3 \mathrm{~mm}$ para o m. masseter de lado esquerdo, o maior valor obtido na mensuração foi de $63 \mathrm{~mm}$ e o menor de $28 \mathrm{~mm}$. A diferença entre as médias do m. masseter direito para o esquerdo foi de $1,3 \mathrm{~mm}$. O resultado da média foi de $44,8 \mathrm{~mm}$ para o $\mathrm{m}$. temporal direito, sendo o maior valor obtido de $64 \mathrm{~mm}$ e o menor de $31 \mathrm{~mm}$. A média para o $\mathrm{m}$. temporal esquerdo foi de $43,8 \mathrm{~mm}$, o maior valor encontrado foi de $62 \mathrm{~mm}$ e o menor de $27 \mathrm{~mm}$. Obteve-se diferença de $1,0 \mathrm{~mm}$ comparando-se o m. temporal direito com o esquerdo. Obteve-se de média para espessura da cabeça da mandíbula do lado direito $57,2 \mathrm{~mm}$, sendo o menor valor encontrado $22 \mathrm{~mm}$ e o maior de $101 \mathrm{~mm}$. Por fim, a média obtida para a cabeça da mandíbula do lado esquerdo foi de $55,4 \mathrm{~mm}$, sendo o maior valor obtido $100 \mathrm{~mm}$ e o menor $23 \mathrm{~mm}$. A comparação das médias obtidas para espessura da cabeça da mandíbula do lado direito e esquerdo resultou em 1,8mm de diferença.

\section{I SCUSSÃO}

Na literatura é comum encontrar estudos utilizando-se de ultrassonografia,

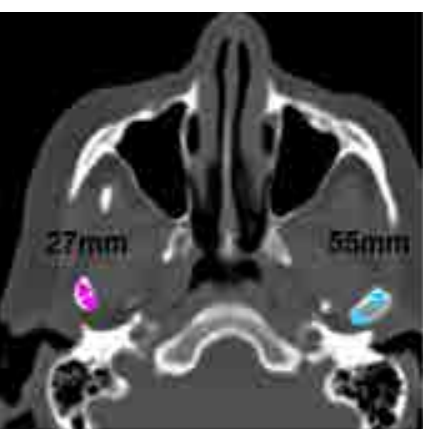

Figura 1: TC - axial, diferença de espessura da CM entre lado direito e esquerdo.

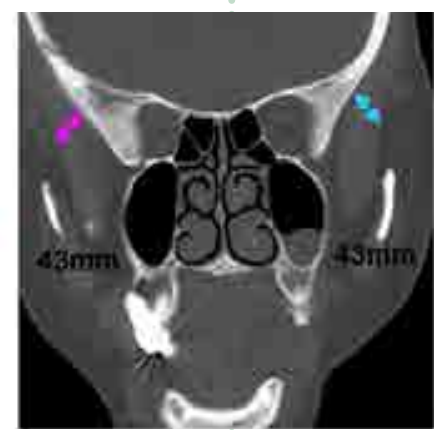

Figura 2: $T C$ - axial, simetria bilateral entre o MT direito e esquerdo.

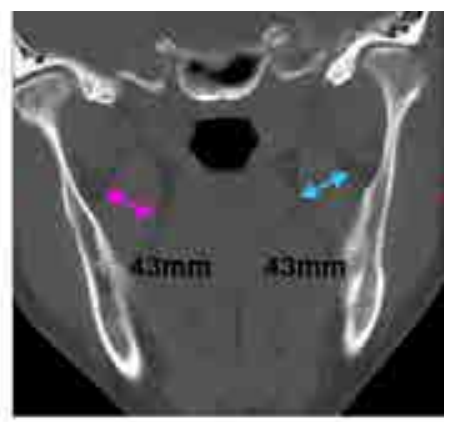

Figura 3: TC - coronal, simetria bilateral entre o MPM direito e esquerdo 


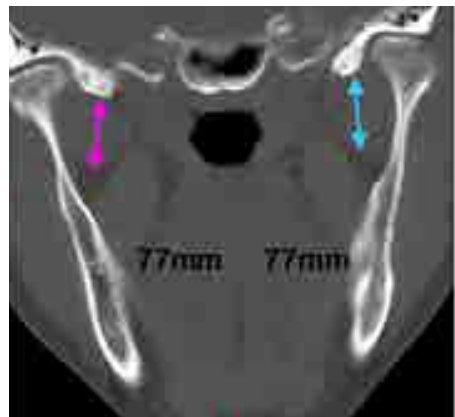

Figura 4: TC - coronal, simetria bilateral entre o MPL direito e esquerdo.

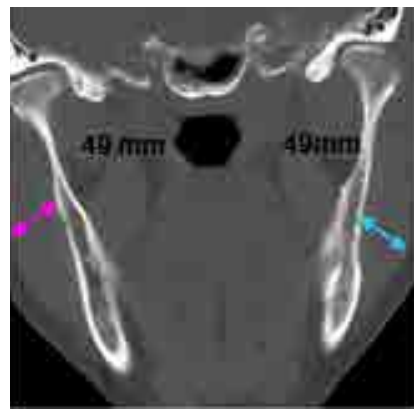

Figura 7: TC - coronal, simetria bilateral entre o MM direito e esquerdo.

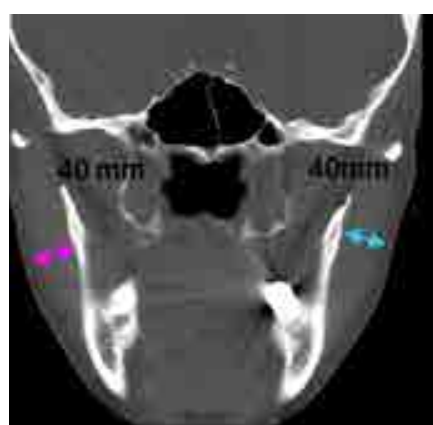

Figura 5: $T C$-coronal, simetria bilateral entre o MM direito e esquerdo.

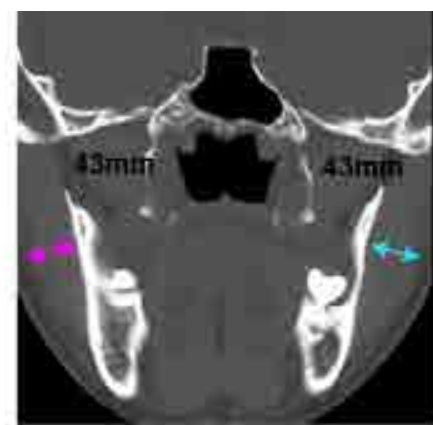

Figura 6: TC - coronal, simetria bilateral entre MM direito e esquerdo.

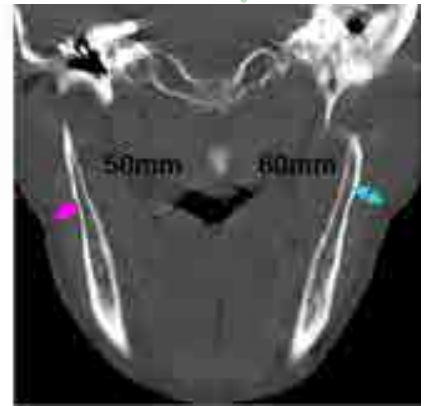

Figura 8: TC-coronal, assimetria entre o MM direito e esquerdo. ressonância magnética e eletromiografia para avaliar a assimetria tanto de tamanho como de atividade muscular no que se refere aos músculos mastigatórios.

Botelho et al. ${ }^{10}$ (2008) realizaram um estudo utilizando eletromiografia referente à assimetria dos m.m. temporal e masseter em pacientes com oclusão normal, dentição permanente completa e sadia, para servir de parâmetro em futuras comparações com sujeitos portadores de DTM. Houve participação de trinta sujeitos no estudo (13 homens e 17 mulheres). Os resultados demonstraram que sujeitos adultos jovens avaliados apresentaram assimetria dentro dos padrões de normalidade já estabelecidos previamente em outras populações no que diz respeito à atividade muscular ${ }^{10}$.

Já D'Ippolito et al. ${ }^{12}$ (2010) realizaram um estudo avaliando a visibilidade do $\mathrm{m}$. pterigóideo lateral na ATM por imagens de ressonância magnética , em diferentes projeções e compararam os achados de imagem com sintomas clínicos de pacien- tes com e sem DTM. Concluíram que na presença de DTM houve alterações na espessura do m. pterigóideo lateral, sendo as principais alterações a atrofia muscular e a contratu$\mathrm{ra}^{12}$.

Comparando a pesquisa realizada com os trabalhos citados, há diferença na metodologia, quantidade da amostra analisada, sujeitos da pesquisa e difere também no que diz respeito a exames clínicos. Já o presente estudo foi realizado com um banco de imagens sem qualquer tipo de pré-requisitos odontológicos para a seleção dos exames utilizados. Sendo assim, a presente pesquisa buscou estabelecer parâmetros, para futuras comparações realizadas em futuros estudos.

\section{CONCLUSÃO}

Os resultados do estudo demonstraram que o m. mais volumoso encontrado foi o pterigóideo lateral do lado direito (70,6mm - média) e o que apresentou menor volume foi o $\mathrm{m}$. temporal esquerdo (43,8mm - média).

Não foi encontrada simetria bilateral na amostra estudada, porém a margem de diferença de espessura dos lados não foi expressiva. Houve equilíbrio quando correlacionados valores da espessura muscular e a espessura da cabeça da mandíbula, ou seja, aqueles que apresentaram maior espessura muscular também apresentaram valores altos de espessura da cabeça da mandíbula. $\angle I C A \quad D P$

TUCUNDUVA MJAPS

ANÁLISE DA

ESPESSURA

DOS MÚSCULOS

MASTIGATÓRIOS

POR MEIO DE

TOMOGRAFIA

COMPUTADORIZADA

HELICOIDAL

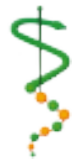

REV. ODONTOL.

UNIV. CID. São

PAulo

2014; 26(1): 188-

95, JAN-ABR 
$\angle I C A \quad D P$

TUCUNDUVA MJAPS

ANÁLISE DA

ESPESSURA

DOS MÚSCULOS

MASTIGATÓRIOS

POR MEIO DE

TOMOGRAFIA

COMPUTADORIZADA

HELICOIDAL

REV. ODONTOL

UNIV, CID, SÃO

PAULO

$2014 ; 26(1): 6-12$

\section{REFERÊNCIAS}

1. Rizzolo RJC, Madeira MC. Anatomia facial com fundamentos de anatomia sistêmica geral. 2. ed. São Paulo: Elsevier; 2006.

2. Okeson J. Tratamento das desordens temporomandibulares e oclusão. 6 . ed. São Paulo: Artes Médicas; 2009.

3. Werlang $\mathrm{H}$, Bergoli $\mathrm{P}$, Madalosso B. Manual do residente de radiologia. Rio de Janeiro: Guanabara Koogan; 2009.

4. Ash MM, Ramfjord S, Schmidseder J. Oclusão. São Paulo: Santos; 2007.

5. Coutinho TA, Abath MB, Campos GJL, Antunes AA, Carvalho RWF. Adaptações do sistema estomatognático em indivíduos com desproporções maxilo-mandibulares: revisão da literatura. Rev soc bras fonoaudiol 2009 14(2):275-9.

6. Felício CM, Mazzetto MO, Bataglion C, Silva MAMR, Hotta TH. Desordem temporomandibular: análise da freqüência e severidade dos sinais e sintomas antes e após a placa de oclusão. J bras ortodon ortop facial 2003 janfev;8(43):48-57.

7. Costa AL, Campos LS, França Jr. MC, D'Abreu A. Temporomandibular disorders in patients with craniocervical dystonia. Arq Neuro-Psiquiatr 2011 Dec;69(6):896-9.
8. Castelo PM, Gavião MBD, Pereira LJ, Bonjardim LR. Avaliação ultra-sonográfica dos músculos mastigatórios e dimensões faciais em crianças com oclusão normal e mordida cruzada posterior unilateral. Rev CEFAC 2007 mar;9(1):61-71.

9. Duarte-Kroll C, Bérzin F, Alves MC. Avaliação clínica da atividade dos músculos mastigatórios durante a mastigação habitual: um estudo sobre a normalização de dados eletromiográficos. Rev odontol UNESP 2010 maiojun;39(3):157-62.

10. Botelho AL, Brochini APZ, Martins MM, Melchior MO, Silva AMBR, Silva MAMR. Avaliação eletromiográfica de assimetria dos músculos mastigatórios em sujeitos com oclusão normal. RFO 2008 set-dez;13(3):7-12.

11. Felício CM, Melchior MO, Silva MAMR, Celeghini RMS. Desempenho mastigatório em adultos relacionado com a desordem temporomandibular e com a oclusão. Pró-Fono $R$ Atual Cient 2007 abr-jun;19(2):151-8.

12. D'Ippolito SM, Borri Wolosker AM, D'Ippolito G, Herbert de Souza B, Fenyo-Pereira M. Evaluation of the lateral pterygoid muscle using magnetic resonance imaging. Dentomaxillofac Radiol 2010 Dec;39(8):494-500. 